\title{
Avaliação não destrutiva de painéis de partículas de média densidade pelo método Stress Wave Timer
}

\author{
Stefânia Lima Oliveira1* ${ }^{1 *}$ Rafael Farinassi Mendes ${ }^{1}$, Lourival Marin Mendes ${ }^{1}$, Carolina Rezende Pinto Narciso ${ }^{1}$, Débora Pereira Ribeiro ${ }^{1}$
}

${ }^{1}$ Universidade Federal de Lavras, Campus Universitário, CP 3037, CEP 37200-000, Lavras, MG, Brasil

"Autor correspondente:

stefaniaoliveira@yahoo.com.br

Termos para indexação:

Bagaço de cana

Eucalyptus

Pinus

Index terms:

Eucalyptus

Pinus

Sugar cane bagasse

Recebido em 07/10/2015

Aprovado em 24/03/2017

Publicado em 29/09/2017

doi: 10.4336/2017.pfb.37.91.1071

\begin{abstract}
Resumo - O objetivo deste trabalho foi verificar a eficiência da avaliação não destrutiva de propriedades mecânicas de painéis de madeira, pelo método de emissão de ondas de tensão (stress wave timer). Foram avaliados painéis MDP industriais, sendo cinco de eucalipto e cinco de pinus, ambos provindos de empresas nacionais, e outros cinco painéis de bagaço de cana importados da China. Para a avaliação não destrutiva dos painéis, foi utilizado o equipamento Metriguard, que utiliza o método de emissão de ondas de tensão Stress Wave Timer. O módulo de elasticidade $\left(\mathrm{MOE}_{\mathrm{d}}\right)$ obtido pelo método de stress wave timer foi confrontado com os valores determinados por meio de ensaios convencionais destrutivos de MOE e módulo de ruptura (MOR) à flexão estática. Em cada painel foram realizadas leituras nas direções longitudinal e transversal dos painéis. O método não destrutivo stress wave timer apresentou eficiência apenas para a utilização em painéis de bagaço de cana, podendo ser utilizado para predição do MOE e MOR desse tipo de painel.
\end{abstract}

\section{Nondestructive evaluation of medium density particleboard by Stress Wave Timer method}

\begin{abstract}
The objective of this study was to verify the efficiency of nondestructive evaluation of wood panels mechanical properties by stress wave timer method in medium density particle board (MDP) from pine. Were evaluated industrial MDP panels from eucalypts (5) and pine (5), both from two national companies, and five panels from sugarcane bagasse imported from China. Stress wave timer method was used for nondestructive evaluation. The modulus of elasticity $\left(\mathrm{MOE}_{\mathrm{d}}\right)$ obtained by stress wave timer method was confronted with MOE and flexural strength (MOR) to bending tests from conventional assays. Measures in length and width directions were performed in each panel. Nondestructive method was effective only for sugar cane bagasse panels, so it can be used to predict MOE and MOR for this type of panel.
\end{abstract}

\section{Introdução}

O setor moveleiro no Brasil apresentou crescimento e desenvolvimento significativo nos últimos anos, sendo composto por pequenas, médias e grandes empresas, as quais têm investido em tecnologia, design e utilização de novos materiais (Oliveira et al., 2016).
Dentre os materiais utilizados para a produção dos móveis nacionais estão os painéis aglomerados do tipo MDP (Medium Density Particleboard). De acordo com dados da Indústria Brasileira de Árvores (IBÁ) (2015), o Brasil demandou de 14,2 milhões de $\mathrm{m}^{3}$ de madeira em tora para a produção de painéis reconstituídos em 2014. 
A caracterização desses painéis é tradicionalmente realizada mediante ensaios destrutivos. No entanto, algumas técnicas baseadas em métodos não destrutivos estão sendo utilizadas para a estimativa de suas propriedades físicas e mecânicas, sem alterar suas características estruturais e seu uso final (Pellerin \& Ross, 2002; Bucur, 2006; Melo \& Del Menezzi, 2016; Gaff et al., 2017). Além de não inutilizar o produto, outro grande diferencial dos ensaios não destrutivos, em relação à caracterização destrutiva, é a rapidez na obtenção da informação (Stangerlin et al., 2010).

Dentre as possibilidades de avalição não destrutiva de painéis está o ensaio através das ondas de tensão (SWT), o qual se baseia na propagação de ondas produzidas pelo impacto de um pêndulo na amostra e o posterior uso do tempo de propagação da onda através da amostra na predição de seu módulo de elasticidade (Bodig, 2001), sendo importante para determinar o quanto o material suporta de esforço sem que ocorram deformações permanentes. Todavia, outros estudos também relacionaram o tempo de propagação de onda com o módulo de ruptura (Han et al., 2006). Em painéis aglomerados, os módulos de ruptura e de elasticidade obtidos pelo ensaio de flexão estática apresentam alta correlação entre si (Protásio et al., 2012).

As duas propriedades são importantes para definição da qualidade do painel para utilização na produção de móveis. Portanto, o uso de métodos não destrutivos para a avaliação de painéis se mostra de grande interesse pela praticidade para a indústria moveleira. Neste contexto, o objetivo deste trabalho foi verificar a eficiência da avaliação não destrutiva através do método de propagação de ondas de tensão (SWT) na predição dos módulos de elasticidade e de ruptura de painéis MDP de pinus, eucalipto e bagaço de cana.

\section{Material e métodos}

Foram adquiridos painéis MDP industriais, sendo cinco de eucalipto e cinco de pinus, advindos de empresas nacionais, e cinco painéis de bagaço de cana, importados da China. As dimensões dos painéis foram de 2,44 x 1,22 x 0,015 m (comprimento x largura $\mathrm{x}$ espessura, respectivamente).

Os painéis foram encaminhados à Unidade Experimental de Produção de Painéis de Madeira (UEPAM), localizada na Universidade Federal de Lavras, Lavras, MG.
O ensaio não destrutivo foi realizado em triplicata nas direções do comprimento e da largura de cada painel inteiro (Figura 1). Para avaliação dos painéis, foi determinado o tempo de propagação de onda entre os dois transdutores do equipamento SWT da marca Metriguard, modelo 239A. O ensaio foi avaliado com umidade relativa média do ar de $72 \%$ e temperatura média de $26^{\circ} \mathrm{C}$.
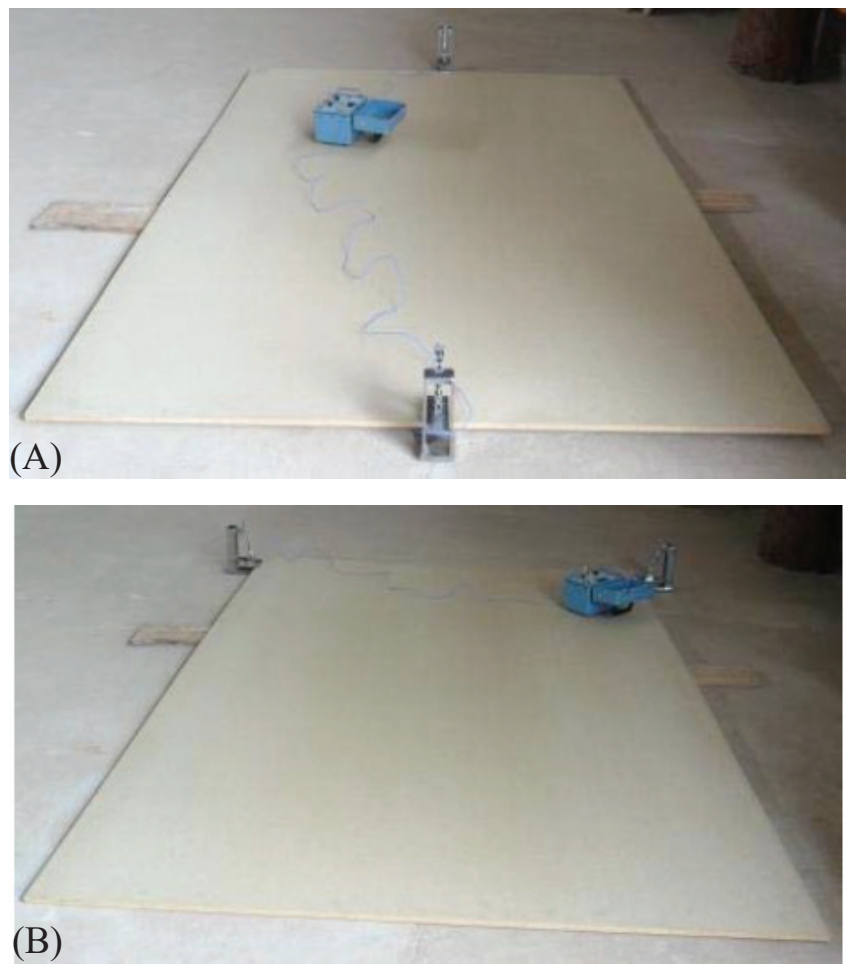

Figura 1. Determinação do $\mathrm{MOE}_{\mathrm{d}}$ pelo SWT no comprimento (A) e largura (B).

A velocidade de propagação das ondas de tensão e o módulo de elasticidade dinâmico foram determinados conforme as equações 1 e 2 , respectivamente.

$$
\begin{aligned}
& V=d / t \\
& M O E d=\text { Me ou } \gamma V^{2}
\end{aligned}
$$

Em que: $\mathrm{V}=$ velocidade de propagação das ondas de tensão $\left(\mathrm{cm} \mathrm{s}^{-1}\right) ; \mathrm{d}=$ distância entre os transdutores $(\mathrm{cm}) ; \mathrm{t}=$ tempo de propagação (s); $\mathrm{MOE}_{\mathrm{d}}=$ módulo de elasticidade dinâmico $\left(\mathrm{kgf} \mathrm{cm}{ }^{-2}\right) ; \mathrm{Me}=$ massa específica do painel $\left(\mathrm{kg} \mathrm{m}^{-3}\right) ; \mathrm{V}=$ velocidade da onda longitudinal $\left(\mathrm{cm} \mathrm{s}^{-1}\right)$.

O módulo de elasticidade obtido pelo método SWT foi confrontado com os valores de módulos de elasticidade (MOE) e de ruptura (MOR) à flexão estática. Para a obtenção de tais propriedades mecânicas, foram utilizados 50 corpos de prova nas dimensões de 250 
x $50 \mathrm{~mm}$ (comprimento x largura, respectivamente), retirados dos centros e bordas e nos sentidos das larguras e comprimentos dos painéis, os quais foram avaliados por meio de ensaios convencionais destrutivos de acordo com a norma NBR 14810-3 (Associação Brasileira de Normas Técnicas, 2006b). Os dados foram analisados utilizando análises de variância e de regressão, ambas a 5\% de significância.

\section{Resultados e discussão}

Os valores médios de densidade aparente encontrados para pinus, eucalipto e bagaço não diferiram estatisticamente (Tabela 1), sendo classificados como painéis de média densidade, que se referem aqueles com densidade aparente entre 0,60 e $0,80 \mathrm{~g} \mathrm{~cm}^{-3}$ (NBR 148101) (Associação Brasileira de Normas Técnicas, 2006a).

Nota-se que houve diferença entre todos os tratamentos para a umidade dos painéis (Tabela 1). Os painéis de pinus apresentaram o maior valor médio de umidade, seguido dos painéis de eucalipto e por último os painéis de bagaço de cana. A norma de comercialização EN 312 (European Committee for Standardization, 1993) estipula a faixa de 5 a $13 \%$ como valores adequados de umidade dos painéis. Sendo assim, todos os tratamentos estão de acordo com o intervalo determinado.

As regressões obtidas para a análise entre o MOE e o $\mathrm{MOE}_{\mathrm{d}}$ e entre o MOR e o $\mathrm{MOE}_{\mathrm{d}}$ estão apresentadas nas Tabelas 2 e 3, respectivamente, para os painéis de pinus, eucalipto e de bagaço de cana.

Na Tabela 1 estão destacados os valores médios de densidade aparente e umidade dos painéis de pinus, eucalipto e bagaço.

Tabela 1. Densidade aparente e umidade dos painéis.

\begin{tabular}{ccc}
\hline \multirow{2}{*}{ Tratamento } & Densidade & Umidade \\
\cline { 2 - 3 } & $\left(\mathrm{g} \mathrm{cm}^{-3}\right)$ & $(\%)$ \\
\hline Pinus & $0,612 \mathrm{~A}$ & $10,9 \mathrm{C}$ \\
Eucalipto & $0,623 \mathrm{~A}$ & $10,0 \mathrm{~B}$ \\
Bagaço & $0,616 \mathrm{~A}$ & $9,0 \mathrm{~A}$ \\
\hline
\end{tabular}

Médias seguidas da mesma letra não diferem entre si pelo teste de Tukey, a $5 \%$ de significância.

Tabela 2. Regressão entre o módulo de elasticidade (MOE) e módulo de ruptura (MOR) d. .

\begin{tabular}{ccccc}
\hline Avaliação & Matéria-prima & Equação & $\mathbf{R}^{\mathbf{2}}$ & Fc \\
\hline \multirow{2}{*}{ Média } & Bagaço de cana & $0,579 \mathrm{x}+1295,7$ & 0,7497 & $8,99 *$ \\
& Eucalipto & $0,266 \mathrm{x}+2286,2$ & 0,2043 & $0,77 \mathrm{~ns}$ \\
& Pinus & $0,1212 \mathrm{x}+2229,5$ & 0,5786 & $4,12 \mathrm{~ns}$ \\
\hline \multirow{2}{*}{ Comprimento } & Bagaço de cana & $0,8424 \mathrm{x}+992,72$ & 0,7341 & $8,28^{*}$ \\
& Eucalipto & $0,4807 \mathrm{x}+1800,6$ & 0,6772 & $6,29 \mathrm{~ns}$ \\
& Pinus & $0,1623 \mathrm{x}+2149,5$ & 0,5208 & $3,26 \mathrm{~ns}$ \\
\hline \multirow{2}{*}{ Largura } & Bagaço de cana & $0,398 \mathrm{x}+1538,9$ & 0,6819 & $6,43^{*}$ \\
& Eucalipto & $0,0268 \mathrm{x}+2911,7$ & 0,0033 & $0,010 \mathrm{~ns}$ \\
& Pinus & $0,0596 \mathrm{x}+2344,1$ & 0,3767 & $1,81 \mathrm{~ns}$ \\
\hline
\end{tabular}

ns: regressão não significativa a 5\% de significância e *regressão significativa a 5\% de significância

Tabela 3. Regressão entre o módulo de elasticidade (MOE) e módulo de ruptura (MOR)

\begin{tabular}{ccccc}
\hline Avaliação & Matéria-prima & Equação & $\mathbf{R}^{\mathbf{2}}$ & Fc \\
\hline \multirow{2}{*}{ Média } & Bagaço de cana & $0,0097 \mathrm{x}-1,5442$ & 0,9346 & $42,89^{* *}$ \\
& Eucalipto & $0,002 \mathrm{x}+12,671$ & 0,4137 & $2,12 \mathrm{~ns}$ \\
& Pinus & $0,0005 \mathrm{x}+12,663$ & 0,7612 & $9,57 \mathrm{~ns}$ \\
\hline \multirow{2}{*}{ Comprimento } & Bagaço de cana & $0,0014 \mathrm{x}-6,5934$ & 0,911 & $30,69^{* *}$ \\
& Eucalipto & $3 \mathrm{E}-0,5 \mathrm{x}+13,077$ & 0,6963 & $6,87 \mathrm{~ns}$ \\
& Pinus & $0,0002 \mathrm{x}+12,353$ & 0,4688 & $2,65 \mathrm{~ns}$ \\
\hline \multirow{2}{*}{ Largura } & Bagaço de cana & $0,0007 \mathrm{x}+2,5361$ & 0,8521 & $17,28^{*}$ \\
& Eucalipto & $0,0001 \mathrm{x}+2,5361$ & 0,224 & $0,86 \mathrm{~ns}$ \\
\hline
\end{tabular}

ns: regressão não significativa a $5 \%$ de significância, ${ }^{*}$ regressão significativa a 5\% de significância e $* *$ regressão significativa a $1 \%$ de significância 
Os painéis de pinus não apresentaram regressão significativa entre as propriedades MOE e o MOR obtido pelo ensaio de flexão estática e o $\mathrm{MOE}_{\mathrm{d}}$ obtido pelo método não destrutivo SWT, independente da direção avaliada.

Freire et al. (2012) avaliaram o módulo de elasticidade dinâmico pelo método não destrutivo SWT em corpos de prova de dimensões de $25 \mathrm{~cm}$, associando os resultados com o módulo de elasticidade obtido por flexão estática em painéis MDP comerciais de pinus. Os autores obtiveram regressão significativa, com $\mathrm{R}^{2}$ de 0,6272. Contudo, o presente trabalho utilizou o próprio painel comercial, o que certamente colaborou para a obtenção de um modelo estatístico não significativo.

Arruda et al. (2011) avaliaram a utilização do bambu gigante para a produção de painéis aglomerados em mistura com Pinus taeda nas porcentagens de 100, 75 e $50 \%$, utilizando adesivo uréia-formaldeído e fenol-formaldeído. Os ensaios não destrutivos foram realizados utilizando o método SWT. A regressão linear entre o $\mathrm{MOE}_{\mathrm{d}}$ e $\mathrm{MOE}$ obtido por ensaio de flexão estática foi significativa em ambos os adesivos, com $\mathrm{R}^{2}$ variando entre 0,66 a 0,91. Han et al. (2006) também obtiveram regressões linear significativas entre o $\mathrm{MOE}$ e o $\mathrm{MOE}_{\mathrm{d}}$ e entre o MOR e o $\mathrm{MOE}_{\mathrm{d}}\left(\mathrm{R}^{2}\right.$ de 0,84 e 0,79, respectivamente), quando avaliaram painéis comerciais aglomerados de pinus, mediante ensaio de flexão estática e pelo ensaio de SWT.

Assim como o observado para os painéis de pinus, os painéis de eucalipto não apresentaram regressão significativa entre o MOE e MOR obtido pelo ensaio de flexão estática e o $\mathrm{MOE}_{\mathrm{d}}$ obtido pelo método não destrutivo SWT.

Mendes et al. (2012), avaliando o módulo de elasticidade de painéis aglomerados de Eucalyptus urophylla por SWT, obtiveram regressão linear simples significativa, com $\mathrm{R}^{2}=0,5936$. Em painéis aglomerados produzidos com madeira de Eucalyptus grandis em associação com bambu nas porcentagens de 0,25, 50, 75 e 100\%, Stangerlin et al. (2010) também obtiveram regressão linear significativa entre o módulo de elasticidade obtido por ensaio de flexão estática e o módulo de elasticidade dinâmico obtido por equipamento de ultrassom, com $\mathrm{R}^{2}=0,5785$.

A não obtenção de regressões significativas entre o MOE e $\mathrm{MOE}_{\mathrm{d}}$ para painéis de pinus e de eucalipto pode ser devido às dimensões industriais dos painéis avaliados, o que faz com que as ondas emitidas encontrem um maior número de espaços vazios no seu percurso e aumente o tempo de propagação, podendo causar assim a diminuição dos valores de correlação com o módulo de elasticidade obtido por ensaio destrutivo (Han et al., 2006).

Diferente do observado para os painéis de pinus e eucalipto, os painéis de bagaço de cana apresentaram relação significativa entre o MOE e MOR obtidos pelo ensaio de flexão estática e o $\mathrm{MOE}_{\mathrm{d}}$ obtido pelo método não destrutivo SWT. De acordo com Vun et al. (2004) e Mendes et al. (2012), a baixa densidade do bagaço de cana, em comparação às madeiras de pinus e eucalipto, permite uma maior compactação das partículas, promovendo a diminuição dos espaços vazios no painel e, consequentemente, uma melhor propagação de ondas emitidas pelo equipamento, permitindo, assim, uma melhor correlação com o MOE e MOR à flexão estática.

Outro fator que ajudou na melhor relação entre o MOE e MOR com o $\mathrm{MOE}_{\mathrm{d}}$ obtido por ensaio não destrutivo é a menor umidade dos painéis de bagaço de cana em relação os painéis de pinus e eucalipto (Tabela 1). De acordo com Almeida et al. (2016), a umidade afeta de forma direta a correlação entre os resultados obtidos pelos ensaios destrutivos e não destrutivos, sendo obtida melhor correlação quanto menor a umidade do material. Han et al. (2006) afirmam que a umidade afeta de forma direta e negativa a velocidade do método stress wave timer, devido a mudança interna do painel.

Vun et al. (2004) avaliaram painéis de bagaço de cana pelo método destrutivo tradicional e pelo método não destrutivo (ultrassom) e obtiveram coeficiente de determinação $\left(R^{2}\right)$ entre o $\mathrm{MOE}_{d}$ e o MOE entre 0,72 e 0,88 , próximo ao valor obtido neste trabalho, que foi de 0,75 .

Mendes et al. (2012), avaliando o efeito de algumas variáveis de produção de painéis aglomerados sobre a relação entre o módulo de elasticidade dinâmico obtido por método não destrutivo SWT com o módulo de elasticidade obtido por flexão estática, obtiveram regressão linear significativa para os painéis de bagaço de cana, com $\mathrm{R}^{2}=0,976$.

Sendo assim, observa-se que o método não destrutivo por SWT pode ser utilizado para predição do MOE e MOR dos painéis de bagaço de cana, tornando-se uma ferramenta prática para a avaliação dos painéis antes da sua utilização, como por exemplo, na produção de móveis. 
Targa et al. (2005) sugerem que, devido ao comportamento viscoelástico, os valores de MOE obtidos nos testes estáticos são inferiores aos dos ensaios dinâmicos. De acordo com Arruda et al. (2011) e Chauhan \& Sethy (2016), as propriedades dinâmicas, normalmente, superestimam as propriedades estáticas e este é o inconveniente da avaliação não destrutiva. No entanto, no presente trabalho não foi observado superestimação dos valores, o que pode estar associado às especificidades de dimensões das partículas, tipo de máteria-prima e dimensões dos painéis utilizados.

\section{Conclusões}

O método não destrutivo SWT apresentou eficiência de utilização apenas para os painéis de bagaço de cana, podendo ser aplicado para predição do MOE e MOR desse tipo de painel.

\section{Referências}

Almeida, D. H. de et al. Determinação da rigidez de Pinus elliottii em diferentes teores de umidade por meio de ensaios mecânicos não destrutivos. Scientia Forestalis, v. 44, n. 110, p. 303-309, 2016. DOI: 10.18671/scifor.v44n110.03.

Arruda, L. M. et al. Lignocellulosic composites from brazilian giant Bamboo (Guadua magna) part 1: Properties of resin bonded particleboards. Maderas Ciencia y Tecnologia, v. 13, n. 1, p. 49-58, 2011. DOI: 10.4067/S0718-221X2011000100005.

Associação Brasileira de Normas Técnicas. NBR 14810-1: chapas de madeira aglomerada: terminologia. Rio de Janeiro, 2006a. 5 p. Parte 1.

Associação Brasileira de Normas Técnicas. NBR 14810-3: chapas de madeira aglomerada: métodos de ensaio. Rio de Janeiro, 2006b.

Bodig, J. The process of NDE research for wood and wood composites. The e-Journal of Nondestructive Testing, v. 6, n. 3, 2001. Disponível em: <http:/www.ndt.net/article/v06n03/bodig/ bodig.htm>. Acesso em: 21 set. 2015. Trabalho apresentado no International Symposium on Nondestructive Testing of Wood, 12., 2001, Sopron.

Bucur, V. Acoustics of wood. 2. ed. Basiléia: Birkhäuser, 2006. 393 p.

Chauhan, S. \& Sethy, A. Differences in dynamic modulus of elasticity determined by three vibration methods and their relationship with static modulus of elasticity. Maderas, Ciencia y Tecnología, v. 18, n. 2, 2016. DOI: S0718-221X2016005000034.
European Committee for Standardization. EN 312: particleboard: specifications. Bruxelas, 1993.

Freire, C. S. et al. Avaliação do módulo de elasticidade dinâmico de diferentes tipos de painéis. In: ENCONTRO BRASILEIRO EM MADEIRAS E EM ESTRUTURAS DE MADEIRA, 13., 2012, Vitória. Anais... Vitória: [s.n.], 2012. CD-ROM.

Gaff, M. et al. Bending characteristics of hardwood lamellae in the elastic region. Composites Part B: Engineering, v. 116, n. 1, p. 6175, 2017. DOI: j.compositesb.2016.12.058.

Han, G. et al. Stress-wave velocity of wood-based panels: effect of moisture, product type, and material direction. Forest Products Journal, v. 56, n. 1, p. 28-33, 2006.

Indústria Brasileira de Árvores. IBÁ: Indústria Brasileira de Árvores. Brasília, DF, 2015. Relatório Ibá 2015. Disponível em: $<$ http://iba. org/images/shared/iba_2015.pdf>. Acesso em: 15 jun. 2017.

Melo, R. R. \& Del Menezzi, C. S. Estimativas das propriedades físico-mecânicas de compostos LVL confeccionados com Paricá por meio de ultrassom. Ciência Florestal, v. 26, n. 1, p. 263-272, 2016. DOI: $10.5902 / 1980509821118$.

Mendes, R. F. et al. Determinação do módulo de elasticidade de painéis aglomerados por Stress Wave Timer. Floresta e Ambiente, v. 19, n. 2, p. 117-122, 2012. DOI: 10.4322/floram.2012.013.

Oliveira, S. L. et al. Particleboard panels made from sugarcane bagasse: characterization for use in the furniture industry. Materials Research, v. 19, n. 4, 2016. DOI: 10.1590/1980-5373-MR-20150211.

Pellerin, R. F. \& Ross, J. R. Nondestructive evaluation of wood. Madison: Forest Products Laboratory, 2002. 210 p.

Protásio, T. P. et al. Correlações entre as propriedades físicas e mecânicas de painéis aglomerados de diferentes espécies de Eucalyptus. Floresta e Ambiente, v. 19, n. 2, p. 123-132. DOI: 10.4322/floram.2012.014.

Stangerlin, D. M. et al. Estimativa do módulo de elasticidade em painéis aglomerados por meio de emissão de ondas ultra-sonoras. Revista Eletrônica de Materiais e Processos, v. 5, n. 3, p. 17-22, 2010.

Targa, L. A. et al. Avaliação do módulo de elasticidade da madeira com uso de método não-destrutivo de vibração transversal. Engenharia Agrícola, v. 25, n. 2, p. 291-299, 2005. DOI: 10.1590/ S0100-69162005000200001.

Vun, R. Y. et al. Through-thickness ultrasonic characterization of wood and agricultural fibre composites. Forest Products Journal, v. 54, n. 12, p. 233-239, 2004. 
